\title{
Correlated Statistical Uncertainties in Coded-aperture Imaging
}

\author{
Matthew C. Fleenor ${ }^{\mathrm{a}, *}$, Matthew A. Blackston ${ }^{\mathrm{b}}$, Klaus P. Ziock ${ }^{\mathrm{b}}$ \\ ${ }^{a}$ Roanoke College, Physics Group, 221 College Lane, Salem, Virginia 24153 \\ ${ }^{b}$ Oak Ridge National Laboratory, P.O. Box 2008, Oak Ridge, Tennessee 37831
}

\section{Abstract}

In nuclear security applications, coded-aperture imagers can provide a wealth of information regarding the attributes of both the radioactive and nonradioactive components of the objects being imaged. However, for optimum benefit to the community, spatial attributes need to be determined in a quantitative and statistically meaningful manner. To address a deficiency of quantifiable errors in coded-aperture imaging, we present uncertainty matrices containing covariance terms between image pixels for MURA mask patterns. We calculated these correlated uncertainties as functions of variation in mask rank, mask pattern over-sampling, and whether or not anti-mask data are included. Utilizing simulated point source data, we found that correlations arose when two or more image pixels were summed. Furthermore, we found that the presence of correlations was heightened by the process of over-sampling, while correlations were suppressed by the inclusion of antimask data and with increased mask rank. As an application of this result, we

\footnotetext{
* Principal corresponding author

Email addresses: fleenor@roanoke.edu (Matthew C. Fleenor), blackstonma@ornl.gov (Matthew A. Blackston), ziockk@ornl.gov (Klaus P. Ziock)
} 
explored how statistics-based alarming is impacted in a radiological search scenario.

Keywords: coded-aperture; uncertainties; detection significance; correlation

\section{Scope, Motivation, \& Focus}

Originally designed as a method to observe high-energy photons in astronomical applications $[1,2]$, coded-aperture imaging is a mature, indirect technique for obtaining spatial information in a variety of imaging applications $[3,4]$. As an elaborate extrapolation from a single pinhole aperture, a mask with structured pattern is placed between a radiation source and a position-sensitive detector, resulting in a modulated hit pattern on the detector $[1,2,3,4]$. The resulting hit pattern is computationally decoded to produce an image of radiation emanating from the field-of-view.

Coded apertures are currently utilized across a spectrum of imaging applications, including astrophysics $[5,6]$ and medicine $[7,8,9]$. The nuclear security community has successfully applied coded-aperture techniques to gamma-ray imaging of radioisotopes for arms control and nuclear nonproliferation applications [10]. In certain cases, the coded-aperture technique provides simultaneously collected spectral information in addition to the spatial location of the sources in question $[4,11]$. Both of these capabilities are valuable to the nuclear community since they provide access to the shape, size, isotopic composition, and activity of the radioisotopes of interest.

In order to be of optimum benefit to the community, quantitative results that rely on statistically meaningful analyses of imaged data are needed. 
However, coded-aperture imaging was designed for point source imaging, and for cases where sources occupy a single pixel, uncertainties are wellknown. Coded-aperture imaging can also be used for extended sources. For point sources that contain strength in adjacent image pixels or for extended sources that cover multiple image pixels, a measure of the total strength of a source requires summing multiple image pixels together. If correlations between image pixels exist, then covariance terms must be properly included in the uncertainty calculation.

The focus of the current study is on the calculation of the uncertainties for the sum of multiple image pixels and these uncertainties as a function of a few important imaging variables. Unlike previous studies, we do not presuppose the absence of correlations when summing multiple image pixels during specific calculations of total source uncertainty. The article maintains the following outline: $\S 2$ introduces the imaging variables manipulated in the current study. In $\S 3$, mathematical formalism of the covariance problem is developed, while $\S 4$ examines image pixel values for degrees of correlation. $\S 5$ presents the degree of correlation as a function of the three image variables, and $\S 6$ discusses applications related to the detection significance within the nuclear security sector.

\section{Coded-Aperture Imaging Variables of Interest}

The imaging variables adjusted in the current work are mask rank, whether or not anti-mask data are included, and mask-pattern oversampling. Each of these variables are important for coded-aperture imaging performed with MURA mask, and may not be applicable to other mask types. A modi- 
fied uniformly redundant array (MURA) is a common mask pattern yielding high signal-to-noise images [12]. They consist of an equal number of open and closed mask elements, giving an open fraction of about $50 \%$.

The rank of the mask, $R$, refers to the prime number on which the MURA pattern is based. Even though other mask pattern arrangements have been found to equal and/or surpass MURA signal-to-noise ratios [13, 8, 14, 15], MURA patterns exhibit ideal imaging properties because the number of artifacts are minimized due to auto-correlative properties [16]. In addition, due to the anti-symmetry of the MURA pattern under rotation, background subtraction is uncomplicated and efficient [17].

The accumulation of anti-mask detector counts afforded by MURA patterns provides a significant advantage for image quality [10]. In rotating an anti-symmetric mask by $90^{\circ}$ and exposing the detector plane, an anti-mask exposure is obtained because the spatial locations of the open and closed mask positions are exchanged $[17,18]$. Subtracting the anti-mask counts from the mask counts suppresses image artifacts and provides an in situ background subtraction [11].

The concept of over-sampling relates to the number of detector pixels that sample each mask opening. In a one-to-one mapping between the detector plane and mask, each opening or closure in the mask is resolved by one detector pixel. This relationship between mask and detector is referred to as single-sampling. When an image is double-sampled, the number of detector pixels covering each mask opening is increased by $2^{2}$ (two times increase in each dimension). The process of over-sampling serves two purposes related to image quality. First, over-sampling aids in the aesthetic problem of pixela- 
tion. For detector planes and instrumentation with an inherently large pixel size, the fourfold increase in the number of image pixels increases the spatial sampling, allowing the shape of objects in the field-of-view to be more easily observed. Second, the number of effective lines-of-sight forming the image increases, which adds to the information present when reconstructing. By remembering that the mask is an effective conglomeration of individual pinholes, each pinhole forms an unique response on the detector (i.e., a basis vector). When the detector plane is over-sampled, there is an increase in the number of basis vectors available to reconstruct the image. However, these basis vectors are not completely independent; rather, they are linear combinations of the original, single-sampled, orthogonal basis vectors.

\section{Correlation \& Covariance within Coded-aperture Imaging}

Since coded-aperture image reconstruction involves a cross-correlation of the detector hit pattern with the MURA mask pattern [3, 12], the value of each reconstructed image pixel $\left(I_{n}\right)$ can be expressed as

$$
I_{n}=\sum_{i} M_{n, i} D_{i}
$$

where $D_{i}$ represents the measured detector counts in pixel $i$, and $M_{n, i}$ is the transfer (or mask) function which is based on the MURA mask pattern with a value of +1 for open mask elements and -1 for closed mask elements (with the exception of the central mask element, which is closed but always set to +1 in order to achieve a delta function response for a point source [19]). Note that in our notation, a single index is used for an image pixel; it should be understood that two-dimensional image pixel indices are collapsed into one 
index for clarity in the expressions that follow. If the detector pixels $\left(D_{i}\right)$ are assumed to have independent errors, i.e., no covariance $\left(\sigma_{D_{i} D_{j}}^{2}=0\right.$, for $i \neq j$ ), the uncertainty on any single image pixel follows as

$$
\sigma_{I_{n}}^{2}=\sum_{i}\left(\frac{\partial I_{n}}{\partial D_{i}}\right)^{2} \sigma_{D_{i}}^{2},
$$

where the partial derivatives are given by the decoding array sampled for a particular image pixel:

$$
\left(\frac{\partial I_{n}}{\partial D_{i}}\right)=M_{n, i}= \pm 1 .
$$

Also since the associated errors for the detector pixels are determined by Poisson statistics (i.e., $\sigma_{D_{i}} \approx \sqrt{D_{i}}$ ),

$$
\sum_{i} \sigma_{D_{i}}^{2}=N
$$

where $N$ is simply the total number of counts in the detector from both source and background. Therefore, the uncertainty on any single-image pixel simplifies to

$$
\sigma_{I_{n}}^{2}=N
$$

i.e., the variance. When including anti-mask data (see $\S 4.1$ ), the total error for single-image pixels will take the form

$$
\sigma_{I_{n}}^{2}=\sum_{i} \sigma_{D_{i}}^{2}=\sum_{i}\left[\left(\sigma_{D_{i}}^{M}\right)^{2}+\left(\sigma_{D_{i}}^{A}\right)^{2}\right]=N^{M}+N^{A}
$$

where the superscripts " $\mathrm{M}$ " and "A" represent the detector counts in a given mask or anti-mask exposure, respectively.

At this point, we may be tempted to naively assume that the total error for the sum of two image pixels is

$$
\sigma_{I_{m}+I_{n}}^{2}=\sigma_{I_{m}}^{2}+\sigma_{I_{n}}^{2}=2 N
$$


However Eq. (6) assumes an independent nature for the image pixel uncertainties and the absence of any correlation between them. Until this assumption can be confirmed for multiple image pixels, we must calculate the covariances between image pixels, namely, $\sigma_{I_{m} I_{n}}^{2}[20]$. Note that in Eq. (1) the vector containing detector counts is the same for each image pixel calculation. The reapplication of the entire dataset to calculate each image pixel value ensures that the image pixel uncertainties will be correlated, which points to the need for the use of the full covariance matrix. Therefore, if two image pixels are summed, the uncertainty for the sum is

$$
\sigma_{I_{m}+I_{n}}^{2}=\sigma_{I_{m}}^{2}+\sigma_{I_{n}}^{2}+2 \sigma_{I_{m} I_{n}}^{2}
$$

Explicitly, the covariance term in Eq. (7) takes the following form:

$$
\sigma_{I_{m} I_{n}}^{2}=\sum_{i}\left[\left(\frac{\partial I_{m}}{\partial D_{i}}\right)\left(\frac{\partial I_{n}}{\partial D_{i}}\right) \sigma_{D_{i}}^{2}\right]+\sum_{i} \sum_{j \neq i}\left[\left(\frac{\partial I_{m}}{\partial D_{i}}\right)\left(\frac{\partial I_{n}}{\partial D_{j}}\right) \sigma_{D_{i} D_{j}}^{2}\right] .
$$

Because we are assuming the detector pixel uncertainties are uncorrelated $\left(\sigma_{D_{i} D_{j}}^{2}=0\right)$, the second term in Eq. (8) goes to zero and the uncertainty for the sum of two image pixels becomes

$$
\sigma_{I_{m}+I_{n}}^{2}=\sum_{i}\left[\left(\frac{\partial I_{m}}{\partial D_{i}}\right)^{2} \sigma_{D_{i}}^{2}+\left(\frac{\partial I_{n}}{\partial D_{i}}\right)^{2} \sigma_{D_{i}}^{2}+2\left(\frac{\partial I_{m}}{\partial D_{i}}\right)\left(\frac{\partial I_{n}}{\partial D_{i}}\right) \sigma_{D_{i}}^{2}\right]
$$

where the first two terms are instances of Eq. (2), and the third term is the remainder of Eq. (8). Having established the values of the partial derivatives as decoder elements of \pm 1 and knowing the detector pixel counts, the uncertainties for image pixel sums are calculable. Further, it is clear from Eq. (9) that the first two partial derivative coefficients are squared, giving always +1 , while the partials in front of the third term could be \pm 1 . To verify the 
mathematical result, an examination of simulated coded-aperture images is now presented.

\section{Evidence of Image Pixel Correlation}

\subsection{Pixel Value Scatter Plots}

One convenient way to visualize correlations between two values is to plot one value versus the other for a large number of independent measurements. In Figure 1, image pixel pairs are plotted for a rank-3 coded aperture with $10^{4}$ distinct simulated measurements. The Upper Left panel of Figure 1 shows a sample simulated image from which the pairs were drawn. The simulations included partial mask transmission and independent Poisson statistics within each detector pixel. Figure 1 (Upper Right) shows an example of nearly uncorrelated pairs of image pixels (e.g., the lower right and lower left image pixels). When one pixel deviates from its mean value in a given direction, there is no directional preference (positive or negative) for the deviation of the other pixel from its mean. The expected uncorrelated distribution is nearly symmetric in both horizontal and vertical directions.

The Bottom panels of Figure 1 show image pixel pairs with observable correlation between them. For image pixel pairs that are positively correlated (Fig. 1, Bottom Left), an increase (decrease) of one pixel from its mean value is typically accompanied by an increase (decrease) of the other pixel. For negatively correlated image pixel pairs (Fig. 1, Bottom Right), an increase (decrease) in pixel value 1 is accompanied by a decrease (increase) in pixel value 2 . It should be noted that the correlations exist regardless of whether the image pixels in question contain the source. We now turn to 
an examination of how a few important variables for coded-aperture imaging (the inclusion of the anti-mask dataset, mask rank, and over-sampling) influence the degree of correlation.

\section{Normalized Covariance}

For all the simulated results shown here, the full covariance was calculated as given in Eq. (9). The diagonal terms (the variances) were equal to the total number of counts in the detector, as given in Eq. (4) for mask-only counts or Eq. (5) for mask+anti-mask counts. In what follows, the covariance matrix was normalized by dividing the maximum value (i.e., the variance, equal for all diagonal elements) into all other terms. This will be referred to as the normalized covariance. In this way, the strength of the covariance was displayed as a fraction of the variance, being either negative or positive.

Depending on the context, Figure 2 displays two convenient ways to show the normalized covariance within a given image. For covariance matrices of moderate ranks, it is straightforward to plot the normalized covariance as a two-dimensional array (Fig. 2, Left). These arrays are constructed by plotting the normalized covariance of each image pixel (indexed on the yaxis) with every other image pixel (indexed on the x-axis). The diagonal terms (i.e., the variance) maintain a value of 1.0, while the off-diagonal terms display fractional values. For covariance matrices of larger ranks, it is helpful to display the normalized covariance of a given image pixel with respect to all other image pixels in image space (Fig. 2, Right). This given image pixel always maintains a value of 1.0 because the covariance of a pixel with itself is simply its own variance. Thus, the image on the right of Fig. 2 is represented 

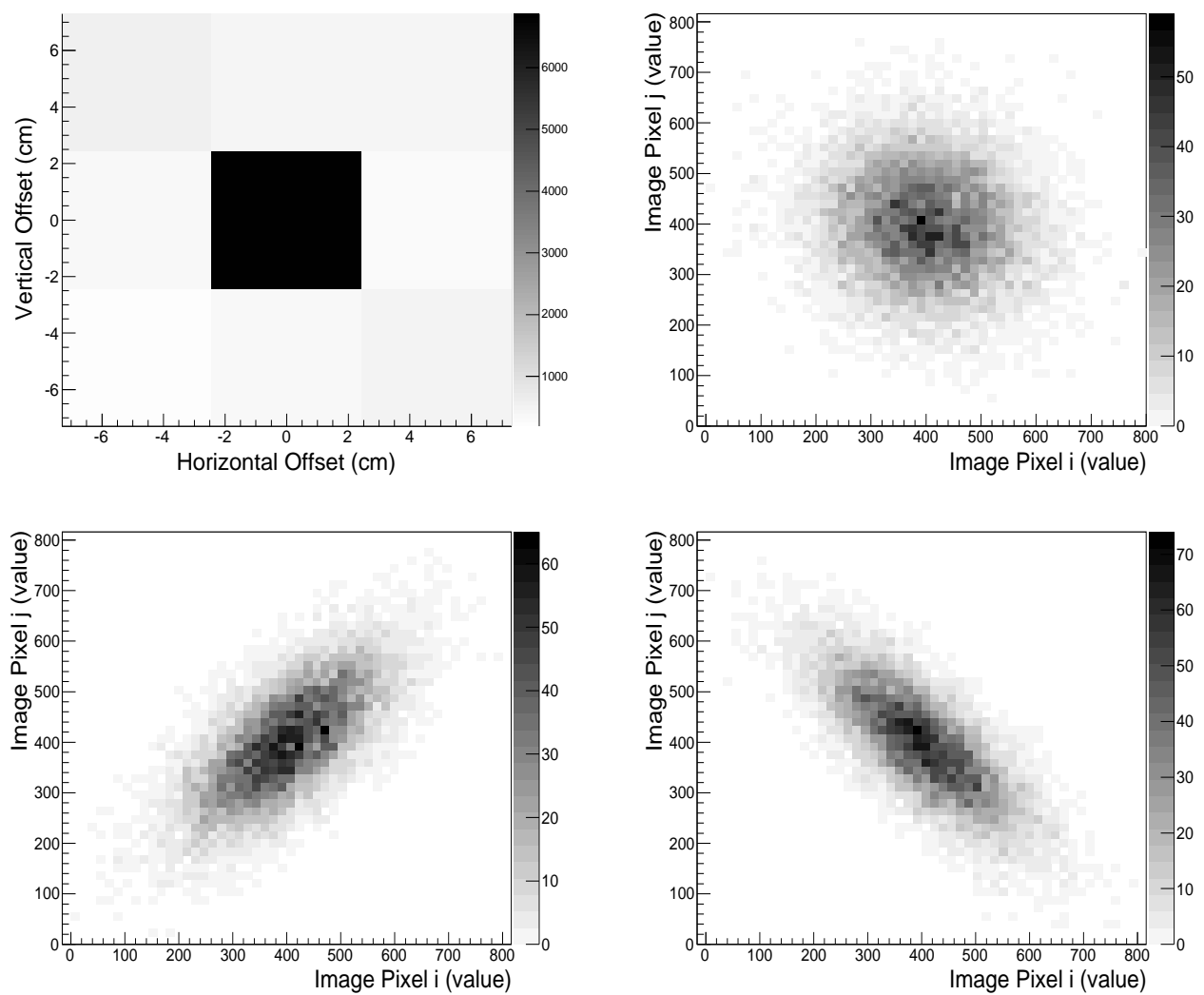

Figure 1: Example simulated rank-3 image, with single-sampling, and image pixel pair scatter plots for $10^{4}$ distinct measurements: (Top Left) example simulated image with centered source (black), one of $10^{4}$, from which the image pixel pairs were taken; (Top Right) an example of a nearly uncorrelated pair of image pixels, taken from the bottom right and bottom left pixels in the images, marked by symmetry about both $\mathrm{x}$ and y axes; (Bottom Left) an example of a positively correlated pair of image pixels, taken from the bottom right and upper left pixels in the images, marked by positive slope; (Bottom Right) an example of a negatively correlated pair of image pixels, taken from the bottom right and middle left pixels in the images, marked by negative slope. 
by one row (or column) in the array display (Fig. 2, Left).
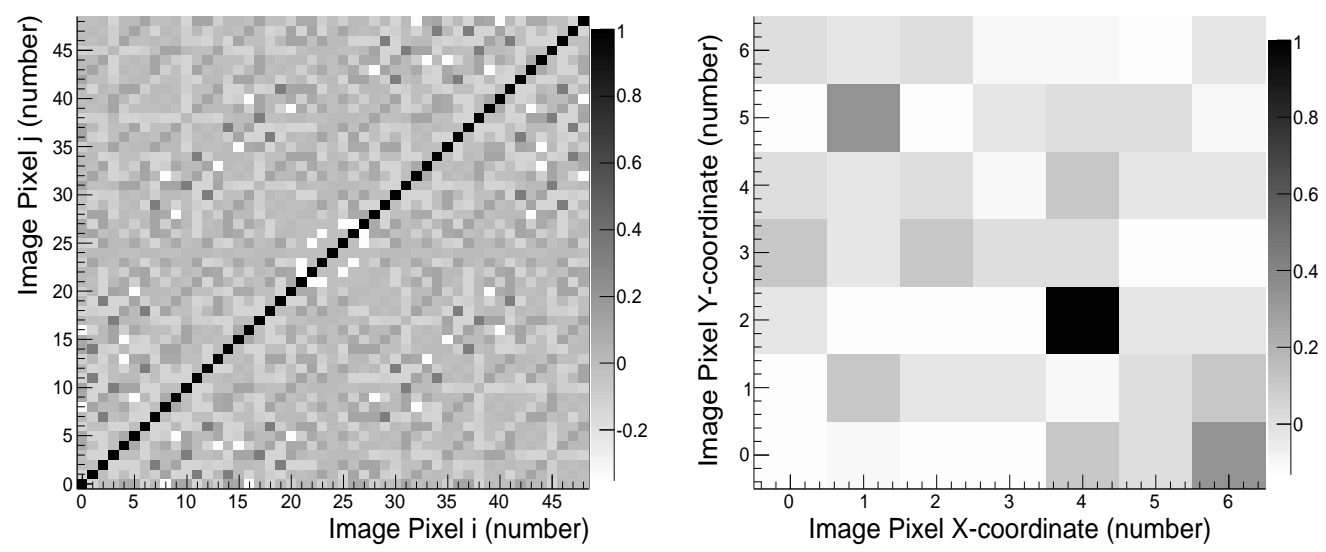

Figure 2: Displays of normalized covariance for a coded-aperture image of rank 7, with single-sampling, and the inclusion of mask counts only: (Left) normalized covariance array displaying the covariance between each pixel pair in the image; (Right) normalized covariance image displaying the covariance between the primary image pixel (equal to 1.0) and every other image pixel. This image corresponds to either Row or Column 19 of the covariance array representation on the left.

\subsection{Covariance as a Function of Anti-Mask Data Inclusion}

Remembering that the anti-mask data are generated by exposing the detector under a $90^{\circ}$ rotation of the MURA mask pattern, the covariance for summed image pixels was examined as a function of including the antimask exposure. Figure 3 shows a normalized covariance array for a rank-7 simulated measurement, with single-sampling, and the inclusion of mask and anti-mask counts (other mask ranks show similar behavior). When compared with Figure 2 (Left), it is clear that reconstructing the image by subtracting 
the anti-mask dataset has advantages for reducing correlations. In what follows, all normalized covariances include mask and anti-mask datasets.

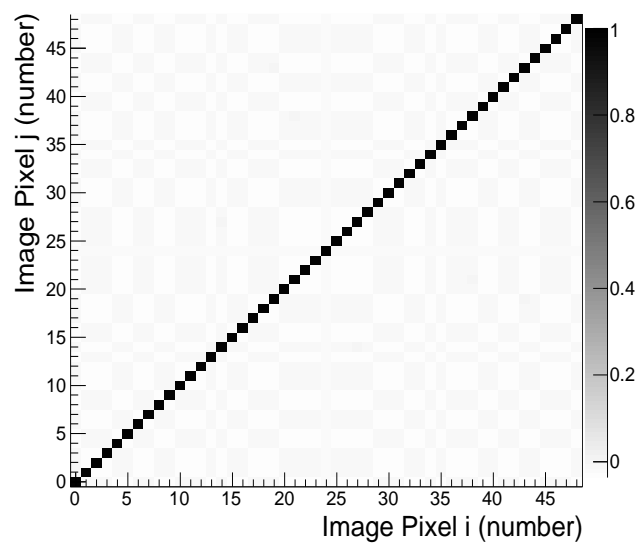

Figure 3: Normalized covariance array for simulated coded-aperture data of rank 7, with single-sampling, and the inclusion of mask and anti-mask datasets. Note that the inclusion of the anti-mask data significantly reduces the variation in the off-diagonal covariance values, bringing them all to nearly zero, when compared with the mask-only array in Fig. 2 (Left). For an explanation of the values in the array, please consult $\S 4$.

\subsection{Covariance as a Function of Mask rank}

The crux of the coded-aperture technique depends on the insertion of a shadow mask with a structured pattern of open and closed elements between the object and detector. The rank of the mask, $R$, refers to the prime number upon which the MURA pattern is based; as the mask rank increases, the number of directions (i.e., the number of image pixels) being imaged also increases. Figure 4 displays the normalized covariance for three different mask ranks, increasing from left to right. All three plots represent single-sampled 


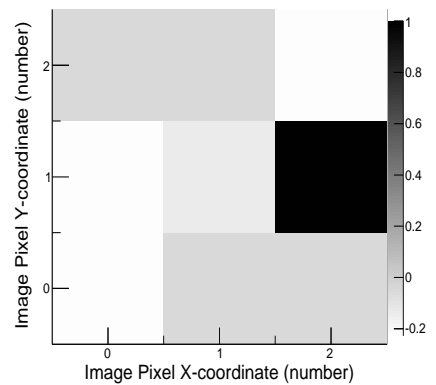
one contribution by $R^{-2}$.

data. This figure shows that increasing the mask rank reduces the covariance, which is not unexpected since the number of detector (and image) pixels has $R^{2}$ dependence, meaning that fluctuations of individual detector pixel values produce less correlation in image pixel values because the overall contribution of any one detector pixel is diminished. During the cross-correlation given by Eq. (1), this increased number of mask elements reduces the effect of any

Figure 4: Normalized covariance images with single-sampling, and the inclusion of mask and anti-mask datasets. These images of normalized covariance show the effect of increasing mask rank, while the image pixel of interest (black) remains in the same general location: (Left) rank-3 mask; (Center) rank-7 mask; (Right) rank-11 mask.
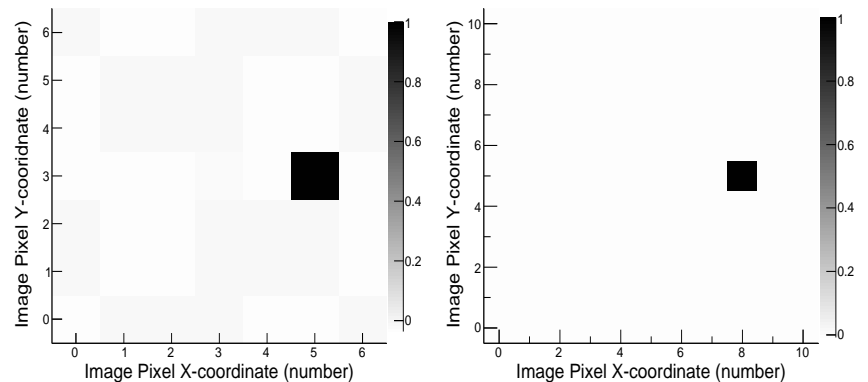

\subsection{Covariance as a Function of Over-Sampling}

The intrinsic angular resolution of a coded-aperture imager is determined by the size of a single mask element divided by the focal length (the distance between the detector and the mask). When there are ample numbers of detector counts, the ability to locate a source can be improved if the amount of radiation through each mask element is sampled more finely than the size of the mask element itself. When more than one detector pixel samples 
a mask opening, the dataset is said to be over-sampled. If the dataset is double-sampled, a $2 \times 2$ array of detector pixels views each mask element.

Figure 5 displays a normalized covariance image of rank 11 with doublesampling, and the inclusion of mask and anti-mask datasets. In comparison to the single-sampled counterpart in Figure 4 (Right), the effects of doublesampling are made clear. The process of double-sampling creates a region of increased (positive) covariance with the nearest neighbors of the selected pixel of interest. In general for masks of higher rank, the perpendicular nearest neighbors contain a normalized covariance of +0.5 , while the diagonal nearest neighbors are +0.25 . Note that the magnitude of the nearest-neighbor covariance does not depend on the presence of a source in either pixel.

While improved ability to localize sources with double-sampled images is desirable, the source strength is spread over multiple pixels and the process of summing multiple pixels introduces covariance. Within the context of statistics-based alarming, one might question whether or not double-sampling is a hindrance due to the introduction of covariance terms. Considering the few cases studied so far, detection significance calculations are similar when comparing images with double-sampling to those with single-sampling. Whether or not alarming sensitivities are reduced or increased by doublesampling more generally, likely depends on several factors (such as where the source is located within an image pixel) that need to be studied.

\section{Impact on Source Detection Significance}

When calculating the statistical significance of a source detection, the detection significance is defined as a ratio of the sum of the image counts in 


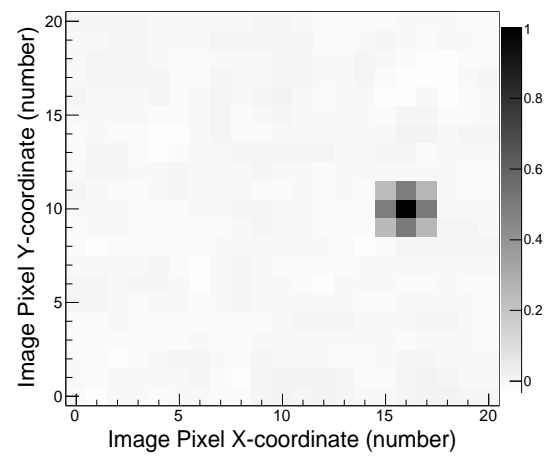

Figure 5: Normalized covariance image of rank 11 with double-sampling, and the inclusion of mask and anti-mask datasets. When compared with the rank-11, single-sampled covariance image in Fig. 4 (Right), the effect of over-sampling is observed. Note that the image with double-sampling shows positive covariance with the nearest eight neighbors to the pixel of interest.

the source region divided by the total uncertainty on that sum, where the resulting quantity is given in units of sigma (e.g., a five-sigma detection). For a single-image pixel, the uncertainty is straightforward and given as the square root of Eq. (3). However, for sources that have strength in multiple pixels, it was demonstrated mathematically and through simulated data that the covariance is not negligible when multiple image pixels are summed. In the following examples, the detection significance is examined in light of the non-zero covariance between summed image pixels.

\subsection{Total Uncertainty of Summed Image Pixels}

In $§ 5.3$ the effect of image over-sampling revealed that the covariance is positive for the eight nearest neighbors of an image pixel. Figure 6 (Left) displays an example image of a double-sampled point source, where the source 
strength is distributed among nine image pixels. When considering the total source strength and its associated uncertainty (i.e., the source detection significance), all nine pixels must be included in the sum. Figure 6 (Right) is a histogram of the sum for those nine pixels for $10^{4}$ distinct simulated images. When only variance terms are used, the calculated expected spread is too narrow and does not correctly predict the observed spread (lighter curve labeled "Variance-only"). However, the calculated expected spread models the observed behavior correctly when covariance terms are included (darker curve labeled "Full Covariance"). This figure demonstrates that the observed spread is accurately predicted only if correlated uncertainties are included. Without accurately calculating these uncertainties, the source detection significance is miscalculated by about $60 \%$.
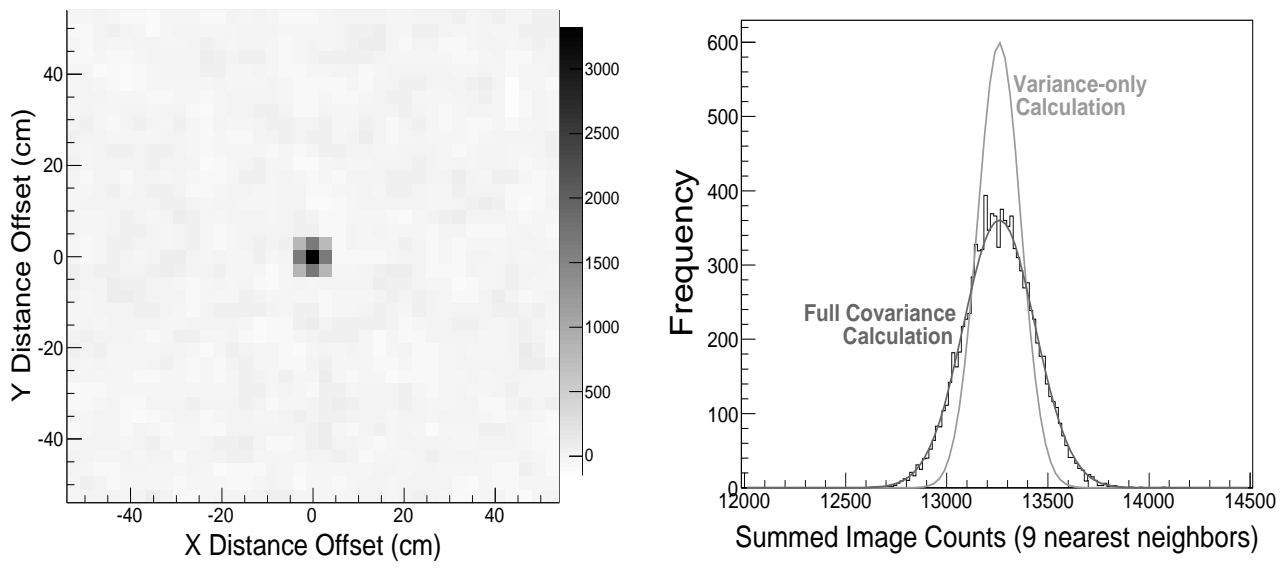

Figure 6: (Left) Example simulated image of a point source for a rank-19 mask, with double-sampling. Note that the values within each image pixel are source counts. (Right) Histogram showing the sum of nine central image pixels for $10^{4}$ simulated images. 
Since the detection significance is given as a ratio of signal over uncertainty, the effect of uncertainty miscalculation has a deleterious effect on the alarm rate. Figure 6 (Right) shows that the total uncertainty is underestimated when only the variance terms are considered. When the alarm threshold is given by the detection significance, an underestimated uncertainty leads to an inflated detection significance and an overconfidence when sounding an alarm. The final section intends to show how this miscalculated uncertainty can lead to false alarms in real-world situations.

\subsection{Detection Alarms \& Coded-aperture Imaging}

Understanding that the correct calculation of the total source uncertainty is intimately connected to alarming, we demonstrate how false alarms could be generated from incorrect calculations of uncertainties in the source. Figure 7 displays measured data from a fast-neutron, coded-aperture imager of mask-rank 11 [21], with double-sampling, and the inclusion of mask and anti-mask counts. The image of the ${ }^{252} \mathrm{Cf}$ source was taken at a mask-source distance of $27 \mathrm{~m}$ and a mask-detector distance of $25 \mathrm{~cm}$. The source extends over four image pixels and is framed by the broken white box (at $\Delta \mathrm{x} \approx 60$ $\mathrm{cm}, \Delta \mathrm{y} \approx 1000 \mathrm{~cm})$. Each individual pixel has a significance near $3 \sigma$ and should be summed to obtain the total strength of the source. When only the variance is considered for the uncertainty on the sum, a value of $190 \pm 30$ counts is calculated. However, when considering the covariance terms for the four image pixels, a value of $190 \pm 40$ counts is calculated. As an example, consider a detection system that maintains an alarm threshold value of $5 \sigma$. The variance-only detection significance equals $190 / 30 \approx 6.3 \sigma$, which would sound an alarm if the threshold were $5 \sigma$. However, the detection significance 
for an uncertainty that incorporates the covariance yields a value of $\approx 4.75 \sigma$ $(190 / 40)$, which would not signal a $5 \sigma$ alarm. This demonstrates how an improper accounting of the total uncertainty for summed image pixels could lead to an excess of false alarms within nuclear security applications.

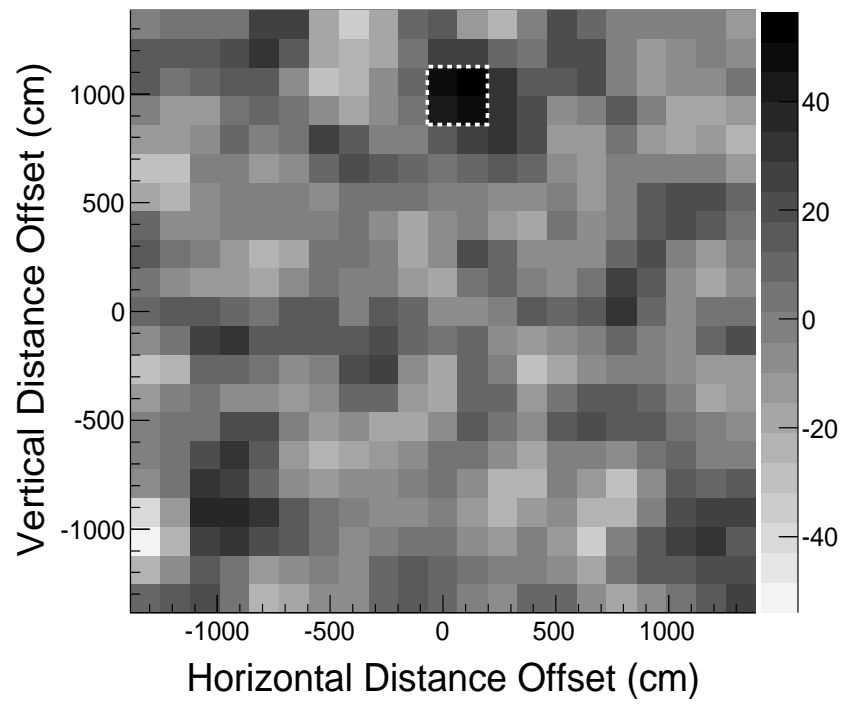

Figure 7: Measured image data for a rank-11, double-sampled coded-aperture image with mask and anti-mask counts. The source extends over four total pixels and is framed by the broken white box (at $\Delta \mathrm{x} \approx 60 \mathrm{~cm}, \Delta \mathrm{y} \approx 1000 \mathrm{~cm}$ ), each with a single pixel significance of $\sim 3 \sigma$. Without the covariance included, the detection significance for the entire extended source would falsely sound an alarm set at $5 \sigma$, see text in $\S 6.2$.

\section{Conclusions}

The current study has demonstrated that correlated uncertainties are reduced in coded-aperture imaging with MURA masks by including the 
anti-mask data and increasing mask rank. However, even when utilizing higher mask ranks and including anti-mask data, correlations between nonneighboring pixels are suppressed but are not entirely negated. Doublesampled data create the largest correlation effects, but almost exclusively in the nearest neighboring pixels (see Fig. 5). This is not unexpected since double-sampling the data breaks the orthogonality of each image pixel with respect to its neighbors. Nearest neighbor correlation exists independently of whether or not source pixels are being examined.

We have calculated the effects of ignoring the covariance when calculating the detection significance, finding that a variance-only uncertainty is narrower than the observed width of simulated data. For these cases, the detection significance is overestimated when the covariance is not incorporated into the uncertainty measurement. The example presented in $\S 5.2$ illustrates how false alarms can arise in radiological search applications if the total uncertainty for summed image pixels is not calculated correctly. This points to the need for the inclusion of covariance terms in situations where oversampling is employed and source strength is summed across multiple image pixels.

\section{Acknowledgments}

We thank the Department of Energy (DOE) for its support of the Visiting Faculty Program (VFP) and Oak Ridge Associated Universities for its administration of the program. We thank Paul Hausladen for helpful discussions. MCF also thanks the Roanoke College Sabbatical Program for its support and the Nuclear Security and Isotope Technology Division for its 
hospitality. This manuscript has been authored by the Oak Ridge National Laboratory, managed by UT-Battelle LLC under Contract No. DE-AC0500OR22725 with the US DOE. The US government retains and the publisher, by accepting the article for publication, acknowledges that the US government retains a nonexclusive, paid-up, irrevocable, worldwide license to publish or reproduce the published form of this manuscript, or allow others to do so, for US government purposes. This work was supported in part by the US Office of Science and the Office of Workforce Development for Teachers and Scientists under the VFP.

\section{REFERENCES}

[1] R. H. Dicke, "Scatter-hole Cameras for X-rays and Gamma Rays", Astrophysical Journal 153 (1968) L101.

[2] J. G. Ables, "Fourier Transform Photography: A New Method for X-ray Astronomy", Proc. Astronomical Soc. Australia 1, 4 (1968) 172.

[3] E. E. Fenimore \& T. M. Cannon, "Coded Aperture Imaging with Uniformly Redundant Arrays", Applied Optics 17, 3 (1978) 337.

[4] J. Gunson \& G. Polychronopolous, "Optimum Design of a Coded Mask X-ray Telescope for Rocket Applications", Monthly Notices Royal Astronomical Soc. 177 (1976) 485.

[5] C. B. Wunderer et al., "Imaging with the Coded Aperture Gamma-ray Spectrometer SPI Aboard INTEGRAL", Proc. SPIE 4851 (2003) 1269. 
[6] J.E. Grindlay \& J. Hong, "Enhanced Wide-field Coded aperture Imaging: Scanning and Radial Holes", Proc. SPIE 5168 (2003) 402.

[7] Zhang, L., Lanza, R. C., Horn, B. K. P., Robert E. Zimmerman, R. E., "Three-dimensional coded-aperture techniques in diagnostic nuclear medicine imaging", Proc. SPIE 3336, Medical Imaging (1998) 1532.

[8] R. Accorsi, F. Gasparini, \& R. C. Lanza, "Optimal Coded Aperture Patterns for Improved SNR in Nuclear Medicine Imaging", Nuclear Instruments \& Methods in Physics Research, Section A (NIM A) 474 (2001) 273.

[9] Starfield, D. M., Rubin, D. M., Marwala, T., "Design of an Ultra-NearField System for Planar Coded Aperture Nuclear Medicine Imaging", Proc. IFMBE 20 (2008) 590.

[10] K. P. Ziock, C. J. Hailey, T. B. Gosnell, J. H. Lupton, \& E A. Harrison "A Gamma-Ray Imager for Arms Control", IEEE Trans. Nuc. Sci. 39, 4 (1992)

[11] K. P. Ziock, M. T. Burks, W. Craig, L. Fabris, E. L. Hull, \& N. W. Madden, "Real-time Generation of Images with Pixel-by-pixel Spectra for a Coded Aperture Imager with High Spectral Resolution", NIM A 505 (2003) 420.

[12] E. E. Fenimore, "Coded Aperture Imaging: Predicted Performance of Uniformly Redundant Arrays", Applied Optics 17, 22 (1978) 3562.

[13] J. J. in't Zand, J. Heise, \& R. Jager, "The Optimum Open Fraction of 
Coded Apertures. With an Application to the Wide Field X-ray Cameras of SAX", Astronomy \& Astrophysics 288 (1994) 665.

[14] G. K. Skinner, "Sensitivity of Coded Mask Telescopes", Applied Optics 47, 15 (2008) 2740.

[15] P. M. E. Shutler, A. Talebitaher, \& S. V. Springham, "Signal-to-noise Ratio in Coded Aperture Imaging", NIM A 669 (2012) 22.

[16] S. R. Gottesman \& E. E. Fenimore, "New Family of Binary Arrays for Coded Aperture Imaging", Applied Optics 28, 20 (1989) 4344.

[17] J. Braga, T. Villela, U. B. Jayanthi, F. D’Amico, \& J. A. Neri, "A New Mask-antimask Coded-aperture Telescope for Hard X-ray Astronomy", Experimental Astronomy 2, 2 (1991) 101.

[18] R. Accorsi \& R. C. Lanza, "Near-field Artifact Reduction in Planar Coded Aperture Imaging", Applied Optics 40, 28 (2001) 4697.

[19] E. E. Fenimore, "Coded Aperture Imaging: The Modulation Transfer Function for Uniformly Redundant Arrays", Applied Optics 19, (1980) 2465.

[20] Bevington, P. R. \& Robinson, D. K. "Data Reduction and Error Analysis for the Physical Sciences, 3rd ed.", McGraw-Hill:New York (2003) 41.

[21] Hausladen, P. A., Newby, J., Liang F., Blackston, M. A., "The Deployable Fast-Neutron Coded-Aperture Imager: Demonstration of Locating One or More Sources in Three Dimensions", ORNL/TM-2013/446 (2013). 